[0212-7199 (2003) 20: 5; pp 247-250] ANALES DE MEDICINA INTERNA Copyright @ 2003 ARAN EDICIONES, S.L

AN MED. INTERNA (Madrid) Vol. 20, N. ${ }^{\circ} 5$, pp. $247-250,2003$

\title{
Enfermedad de Kikuchi: a propósito de un caso y revisión de la literatura
}

\author{
M. A. LOZANO PARRAS, P. ANGUITA ALONSO, R. CIGÜENZA GABRIEL, \\ E. CALVO MANUEL, J. ALBA LOSADA, D. ESPINÓS PÉREZ \\ Servicio de Medicina Interna I. Hospital Clínico San Carlos. Madrid. ${ }^{\text {SServicio de }}$ \\ Anatomía Patológica. Complejo Hospitalario Xeral Calda. Lugo
}

KIKUCHI'S DISEASE: A CASE REPORT AND LITERATURE REVIEW

\section{RESUMEN}

La enfermedad de Kikuchi-Fujimoto, también conocida como linfadenitis necrotizante histiocitaria no linfocítica, es una entidad clínica poco frecuente que afecta principalmente a mujeres jóvenes de procedencia asiática, aunque tiene una distribución universal. Es característica la aparición de fiebre y adenopatías, habitualmente localizadas a nivel cervical, aunque hay casos descritos de afectación generalizada o extraganglionar.

Su interés estriba en que, aún siendo un proceso benigno y autolimitado, es necesario un diagnóstico anatomo-patológico de exclusión de otras entidades clínicas de peor pronóstico que precisan tratamiento más específico (linfoma, histiocitoma...).

A pesar de que su etiopatogenia es desconocida, se postula un origen vírico y una mediación inmunológica en el desarrollo de la enfermedad. Se ha descrito asociación de la enfermedad de Kikuchi con procesos como el lupus eritematoso subcutáneo, tiroiditis/ Hashimoto, lo que obliga a un posterior seguimiento inmunológico de la enfermedad tras su diagnóstico, ante la posibilidad de aparición con el tiempo de un proceso autoinmune.

Se presenta el caso de una enfermedad de Kikuchi asociada a tiroiditis subaguda linfocitaria.

PALABRAS CLAVE: Enfermedad de Kikuchi. Linfadenitis necrotizante. Linfadenopatías cervicales. Tiroiditis subaguda.

\begin{abstract}
Kikuchi-Fujimoto disease, also known as hystiocytic necrotizing lymphadenitis, is an unusual entity which affects predominantely young asiatic females, although its distribution is world-wide. Cardinal symptoms are fever and adenopathy, generally cervical, although generalized and extraganglionar cases have been described.

Considered a self-limiting disease, it has to be differentiated by pathologic analysis from other less-benign disorders such as lymphoma or hystiocitoma, which bear worse prognosis and may require specific treatment.

Although the origin of this disease is unknown, a viral origin is postulated and the inmune system is involved. Kikuchi disease has been associated to other entities such as subcutaneous lupus erythematosus, Hashimoto thyroiditis, etc...requiring patients be followed inmunologically after diagnois of this disease.

We present a case of Kikuchi disease associated with subacute lymphocytic thyroiditis.
\end{abstract}

KEY WORDS: Kikuchi disease. Necrotizing lymphadenitis. Cervical lymphadenopathy. Subacute thyroiditis.

Lozano Parras MA, Anguita Alonso P, Cigüenza Gabriel R, Calvo Manuel E, Alba Losada J, Espinós Pérez D. Enfermedad de Kikuchi: a propósito de un caso y revision de la literatura. An Med Interna (Madrid) 2003; 20: 247-250.

\section{INTRODUCCIÓN}

Inicialmente descrita en Japón en 1972 por Kikuchi y posteriormente por Fujimoto, es una enfermedad de distribución universal, aunque continúa siendo más frecuente en los países asiáticos. No existen datos sobre la prevalencia en nuestro país, habiéndose publicado tan sólo estudios de casos. Se observa principalmente en mujeres jóvenes, y cursa con la aparición de linfadenopatías, dolorosas o no, habitualmente localizadas a nivel cervical, aunque pueden afectar a las cadenas submandibulares, supraclaviculares, mesentéricas, axila- res e inguinales. Las adenopatías suelen ser de consistencia elástica y variables en número y tamaño. Acompañando al cuadro, es característica la presencia de fiebre, sudores nocturnos y un buen estado general.

\section{CASO APORTADO}

Presentamos el caso de una mujer de 26 años, sin antecedentes de interés que, encontrándose previamente bien, comienza con un cuadro de odinofagia leve, fiebre vespertina de hasta $38,5^{\circ}$ que cede bien con paracetamol, y malestar general. No escalofríos ni crisis

Trabajo aceptado: 27 de marzo de 2002

Correspondencia: M. A. Lozano. C/ Leñeros, 44, 1º-F. 28039 Madrid. 
sudorales acompañantes. 4-5 días después, nota la aparición de ganglios en ambas zonas laterocervicales por lo que inicia tratamiento con amoxicilina, $500 \mathrm{mg} / 8 \mathrm{~h}$, por cuenta propia sin observar mejoría.

En la exploración física destacaba la presencia de adenopatías en ambas cadenas laterocervicales, de consistencia media, rodaderas, no adheridas, de 1-2 cm de diámetro e indoloras. No se palparon adenopatías a otros niveles ni tampoco organomegalias. No bocio ni dolor a la presión sobre la región tiroidea.

El estudio analítico mostró leucocitosis de predominio linfocítico, con una VSG de $22 \mathrm{~mm} / \mathrm{h}$. La función hepática estaba conservada, no existía hipergammaglobulinemia y el mantoux fue negativo. La radiografía de tórax resultó normal. La serología para los virus estudiados fue negativa (anti-EBV, CMV, VIH, HTLV-1, HV-6, toxoplasma y lúes). En el estudio inmunológico: ANA: 1/40 (80), antiDNA: 13,4 UI/ml (50), antiRNA: negativo, ENA-RNP: 0,45 y anticardiolipina: negativo. Se valoró la función tiroidea: T3: 1,31 mg/L (0,6-1,6), T4: 84 mg/L (46-93), TSH: 6,3 mU/L (<7), TGB (antitiroglobulina): 20,17 U/mL (<60), TPO (antiperoxidasa): 1071 $\mathrm{U} / \mathrm{mL}$. La gammagrafía tiroidea fue normal.

La fiebre desapareció a los 12 días, no así las adenopatías que permanecieron sin cambios, por lo que se procedió a la realización de biopsia de dos ganglios de la zona media de la cadena latero-cervical derecha, que resultó diagnóstica. El examen histológico (Figs 1-3) fue compatible con el de Linfadenitis necrotizante histiocitaria no linfocítica o Enfermedad de Kikuchi-Fujimoto.

En el control evolutivo de la función tiroidea a los 5 meses de iniciado el cuadro, se observó un descenso en el título de los anticuerpos antitiroideos (TGB: 7,6 U/mL; TPO: $870 \mathrm{U} / \mathrm{mL}$ ), manteniendo cifras normales de hormonas tiroideas así como de TSH $(2,95$ $\mathrm{mU} / \mathrm{L})$. Nuestro diagnóstico fue de tiroiditis subaguda linfocitaria asociada a enfermedad de Kikuchi.

\section{DISCUSIÓN}

La enfermedad de Kikuchi es un proceso que afecta sobre todo a mujeres jóvenes, por debajo de los 30-40 años, siendo la aparición de adenopatías cervicales la forma de presentación más frecuente, aunque puede afectar a cualquier cadena ganglionar (submandibular, supraclavicular, mesentérica, axilar e inguinal) (1-8), habiéndose descrito ocasionalmente también formas generalizadas (9). La fiebre es el síntoma que más frecuentemente acompaña al cuadro, aunque puede faltar en un $50 \%$ de los casos.

No obstante, hay datos recogidos en la literatura de afectación extraganglionar, habiéndose observado la presencia de un rash cutáneo en un $30 \%$ de los casos $(10,11)$, artralgias migratorias $(12,13)$, sudores nocturnos, pérdida de peso y dolor abdominal secundario a la presencia de adenopatías mesentéricas o a la ocasional hepato-esplenomegalia que pueden tener algunas pacientes $(14,16)$. De forma puntual están descritos casos de meningitis linfocitaria benigna (17), hipertensión intracraneal con edema cerebral secundario a obstrucción del retorno venoso, rabdomiolisis severa (18) e, incluso, un caso de muerte por cardiopatía en un hombre joven (no establecida una clara relación) (19).

La etiopatogenia de esta enfermedad es realmente desconocida. Por un lado se postula una naturaleza vírica apoyada tanto por hallazgos serológicos como ultraestructurales. Se ha descrito la enfermedad de Kikuchi asociada a: EBV (20-21), CMV, HV-6 (22,23), HV-8 (Kaposi) (24), HTLV-1 (25), HIV (26,27), parvovirus B- ${ }_{19}$ (28), parainfluenza, toxoplasma (29), yersinia enterocolítica (30) y brucelosis (31).
Dentro de los hallazgos ultraestructurales, se ha encontrado un aumento de IFN- $\alpha$ en el citoplasma de las células histiocíticas, así como de la 2-5 oligoadenilato sintetasa, enzima citosólica inducida por el IFN- $\alpha$ que aumenta por tres veces sus cifras basales en respuesta a una infección viral aguda. El hallazgo de estructuras tubulo-reticulares (por microscopía electrónica) en el interior de los histiocitos, linfocitos activados y las células endoteliales de los capilares, habla igualmente a favor del posible origen vírico de la enfermedad, dato también relacionado con la producción de IFN- $\alpha(32,33)$.

Por otro lado, se postula una mediación inmunológica, observándose una alteración de la inmunidad celular (linfocitos T) con hipersensibilidad retardada a algunos antígenos causales (34). Existe una activación policlonal de los linfocitos T (CD4 y CD8) que desaparece en la fase de recuperación, un aumento de los monocitos y una ausencia de polimofonucleares neutrófilos, probablemente por secreción de alguna linfocina por los linfocitos activados. Se ha descrito asociación de la enfermedad de Kikuchi con: LES $(35,36)$, enfermedad de Still del adulto (37,38), tiroiditis/Hashimoto $(39,40)$, trasplante hepático y en relación con escapes de implantes mamarios de silicona (41). En nuestro caso, el proceso relacionado consistió en una tiroiditis subaguda linfocitaria, muchas veces de curso asintomático y de diagnóstico casual (42).

El diagnóstico diferencial de la enfermedad de Kikuchi se plantea con otras causas de adenopatías que agrupamos en cuatro grandes campos: enfermedades infecciosas, autoinmunes, onco-hematológicas y otras enfermedades de origen incierto (Tabla I) (43-46).

El diagnóstico de la enfermedad es anatomo-patológico, observándose zonas de necrosis paracortical rodeadas de agregados histiocitarios. A pequeño aumento, los nódulos linfáticos se aprecian típicamente moteados, a causa de las múltiples y a veces coalescentes zonas pálidas rodeadas de otras áreas más oscuras de hiperplasia paracortical. La afectación es característicamente parcheada pudiendo localizarse tan sólo en uno de los ganglios estudiados (Fig 1). A mayor aumento vemos que las zonas pálidas corresponden a áreas de necrosis eosinófila granular con detritus celulares, rodeados de células histiocíticas, células plasmocitoides e inmunoblastos (Fig 2). Hay histiocitos con formas fagocíticas de núcleo excéntrico (imagen típica en "media luna") con presencia de estructuras tubulo-reticulares en su citoplasma (iguales a las observadas en el LES) e histiocitos no fagocíticos de núcleo central con ausencia de detritus celulares en su citoplasma. Es característica la ausencia de polimofonucleares en esta enfermedad.En las zonas de hiperplasia paracortical se puede apreciar una apariencia en "cielo estrellado" por los abundantes linfocitos pequeños entremezclados con inmunoblastos e histiocitos. Estos linfocitos son predominantemente linfocitos $\mathrm{T}$ a expensas de CD8, muy escasos CD4, y algún linfocito B aislado (47-49).

Se han encontrado fenómenos de apoptosis en las células necróticas, comprobado por la presencia de perforinas, proteína citolítica esencial para la misma, y de un gránulo citotóxico propio de las células NK y de los linfocitos T, el TIA1 (50).

Los hallazgos histológicos marcan los estadíos evolutivos de la enfermedad. Existe una primera fase proliferativa de predominio del componente reactivo. Es en este momento, 


\begin{tabular}{|c|c|}
\hline \multicolumn{2}{|c|}{ TABLA I } \\
\hline \multicolumn{2}{|c|}{$\begin{array}{c}\text { DIAGNÓ STICO DIFERENCIAL ENTRE DISTINTAS CAUSAS } \\
\text { DE ADENOPATÍAS }\end{array}$} \\
\hline ENFERM EDADES INFECCIOSAS & ENFERM EDADES ONCO \\
\hline Faringitis estreptocócica & -HEMATO LÓGICAS: \\
\hline Difteria & Cáncer (metástasis) \\
\hline Tularemia & LLC \\
\hline Yersinia & Leucemia aguda \\
\hline Enfermedad por arañazo del gato & Linfomas: \\
\hline Enfermedad de Whipple & Enfermedad de Hodgkin \\
\hline Brucelosis & LNH \\
\hline Fiebre tifoidea & Linfoma Burkitt \\
\hline Leptospira & Linfoma linfoblástico \\
\hline Dengue & Linfoma de células T \\
\hline Listeria & Linfoadenopatía \\
\hline Enfermedad de Lyme & angioinmunoblástica \\
\hline Tuberculosis (escrófula) & Enfermedad de Castleman \\
\hline Lúes & Histiocitosis maligna \\
\hline Chancroide & Histiocitosis de células de \\
\hline Linfogranuloma venéreo & Langerhans \\
\hline VIH & Enfermedad de Rosai-Dorfman \\
\hline $\begin{array}{l}\text { EBV/ CM V / HSV } \\
\text { Enfermedades exantemáticas }\end{array}$ & Enfermedad de Kikuchi-Fujimot \\
\hline \multicolumn{2}{|l|}{ Toxoplasma } \\
\hline \multicolumn{2}{|l|}{ Histoplasma/Criptococo } \\
\hline Infecciones cutáneas & $\begin{array}{l}\text { ENFERM EDADES DE ORIGEN } \\
\text { INCIERTO }\end{array}$ \\
\hline ENFERM EDADES AUTO INM UNES & Fiebre mediterránea familiar \\
\hline LES & Amiloidosis \\
\hline Artritis Reumatoide & Trasplante de silicona \\
\hline $\begin{array}{l}\text { Enfermedad de Still del adulto } \\
\text { Síndrome de Sjögren }\end{array}$ & $\begin{array}{l}\text { Fármacos (fenitoína, quinidina, } \\
\text { enfermedad del suero) }\end{array}$ \\
\hline Enfermedad de Kawasaki & Hiperinmunoglobulinemia D \\
\hline Sarcoidosis & Síndrome PFAPA (fiebre periódica, \\
\hline Enfermedad mixta del tejido conectivo & $\begin{array}{l}\text { adenitis, faringitis y estomati- } \\
\text { tis aftosa) }\end{array}$ \\
\hline
\end{tabular}

VIH: virus de la inmunodeficiencia humana; EBV: virus de Ebstein-Barr; CM V: citomegalovirus; HSV: virus herpes simple; LES: lupus eritematoso sistémico; LLC: leucemia linfática crónica; LNH: linfoma no Hodgkin.

cuando el proceso de necrosis es limitado y los linfoblastos dominan el cuadro, donde es más difícil el diagnóstico diferencial con el linfoma maligno $(51,52)$. A continuación el cuadro evoluciona a una segunda fase necrotizante de necrosis coagulativa, con posterior fase xantomatosa con predominio de histiocitos espumosos y una última de reestructuración de la arquitectura ganglionar y reabsorción del material necrótico.

La evolución benigna de la enfermedad de Kikuchi determina un cuadro autolimitado, con resolución espontánea en 2-

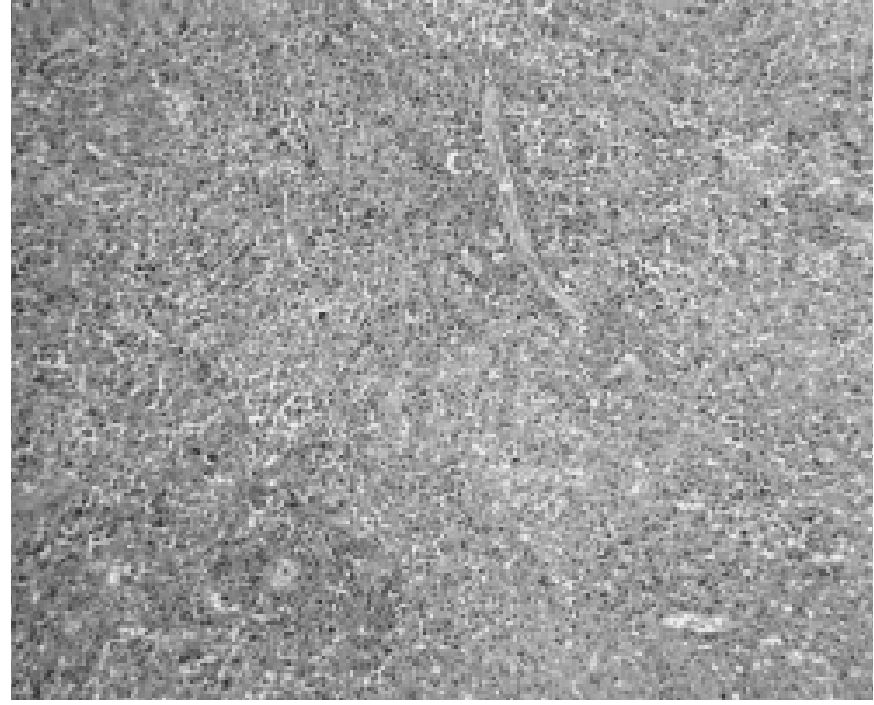

Fig. 1. Imagen parcheada del ganglio. Las áreas claras corresponden a necrosis no neutrofílica (apoptosis). En las áreas más oscuras abundancia de inmunoblastos y plasmablastos. (HE.100X).

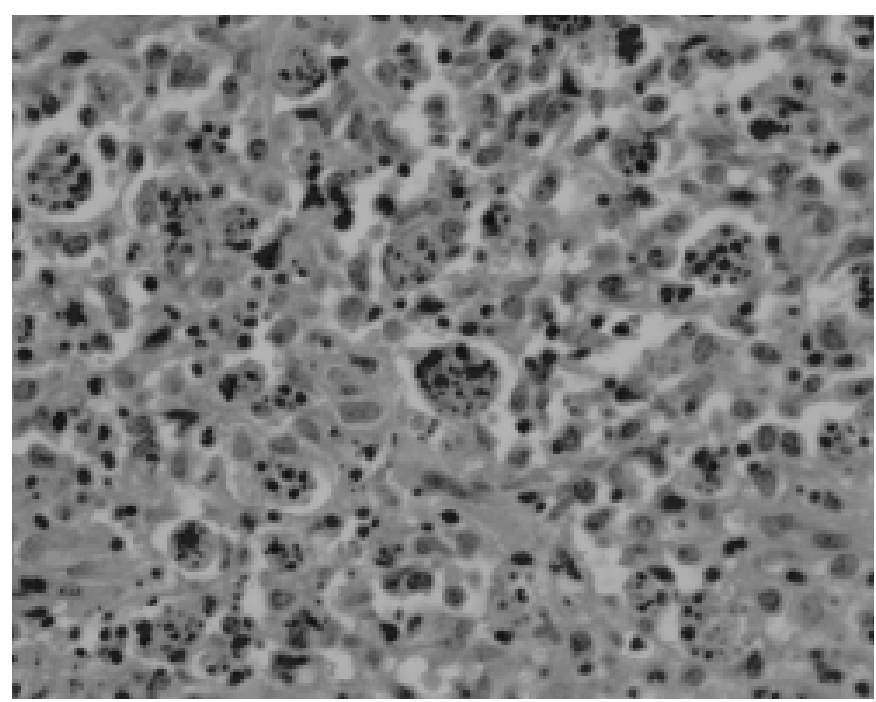

Fig. 2. Abundantes restos necroapoptóticos en ausencia de polimorfonucleares. En el cuadrante superior derecho inmunoblastos y plasmablastos rodean a una vénula epitelioide. (HE.630X).

3 meses. Ocasionalmente se han visto formas recurrentes en un 3-5\% (54), y formas complicadas de posterior evolución a LES. Esto obliga, ante el diagnóstico de la enfermedad, a un posterior seguimiento inmunológico por la posible aparición en el tiempo del proceso autoinmune. Hay casos descritos en la literatura de evolución fatal en la enfermedad de Kikuchi (una muerte por cardiopatía y tres en enfermos transplantados) (54-56).

El tratamiento de la enfermedad es púramente sintomático. Hay un caso descrito de enfermedad de Kikuchi asociado a brucelosis en el que el tratamiento de la enfermedad de base (doxiciclina más estreptomicina) produjo la curación del cuadro.

En casos complicados con ANA positivo y aumento importante de LDH, se ha recomendado el uso de inmunosupresores o corticoides para prevenir un resultado fatal. 


\section{Bibliografía}

1. Álvarez Alegret R, Sanz Moncasi MP. Linfadenitis histiocítica necrotizante de Kikuchi-Fujimoto: ¿Enfermedad o síndrome? Sangre 1996; 44: 337-9.

2. Torres M, Masiá M, Gómez A, Gutiérrez F, Benito C, Vilar A. Linfadenitis histiocítica necrotizante (enfermedad de Kikuchi-Fujimoto): aportación de dos casos. Rev Clin Esp 1996; 196: 706-9.

3. Nikanne E, Roupi P, Vornanen M. Kikuchi's Disease: Report of three cases and o overview. Laryngoscope 1997; 107: 273-6.

4. Zabaleta M, Mollá FJ, Martínez MI, Sánchez L, Erdozain I. Enfermedad de Kikuchi. Acta Otorrinolaring/ Esp/ 1997; 48: 322-4.

5. Nieman RB. Diagnosis of Kikuchi's disease. Lancet 1990; 335: 295.

6. Lorenzo A, Gamallo C, Ortega L y cols. Linfadenitis necrotizante histiocitaria: enfermedad de Kikuchi-Fujimoto. A propósito de 6 casos. An Med Interna (Madrid) 1999; 16; 301-4.

7. Morillas PJ, González MA, Ferrandis E, Serrano E, Guallart F, Ferrer R. Acta Otorrinolaring. Esp; 47: 247-250.

8. Martínez-Vázquez CA , Bordon J, Rubianes M y cols. Enfermedad de Kikuchi. Un estudio clínico-patológico de tres casos. An Med Interna (Madrid) 1998; 15: 367-9.

9. Abba AA, Afzal M, Almoharab FI y Baez-Giangreco A. Kikuchi's disease presenting as bilateral hilar lymphadenopathy. Respiratory Medicine 1995; 89: 701-3.

10. Spies J, Foucar K, Thompson CT and LeBoit PE. The histopathology of cutaneous lesions of Kikuchi's disease (Necrotizing Limphadenitis). Am J Surg Pathol 1999; 23: 1040-7.

11. Kuo TT. Cutaneous manifestation of Kikuchi' necrotizing lymphadenitis. Am J Surg Pathol 1990; 14: 872-6.

12. Moraes MF, da Graca JP, Araujo C, DE Matos AA, de Lacerda MJ, Abecasis P. Kikuchi's disease. A rare cause of fever, lymphadenopathies and polyarthritis. Acta Med Port 1997; 10: 103-6.

13. Heldenberg D, Amar M, Ben-Arie Y, Iuchtman M. Axillary involvement in pediatric Kikuchi's disease. Eur J pediatr Surg 1996; 6: 32-4.

14. Rudniki C, Kessler E, Zarfati M, Turani H, Bar-Ziv Y, Zahavi I. Kikuchi's necrotizing lymphadenitis. A cause of fever of unknown origin and splenomegaly. Acta Hematol 1988; 79: 99-102.

15. Bailey EM, Klein NO, Cuhcha GA. Kikuchi's disease with liver dysfunction presenting as fever of unknown origin. Lancet 1989; 2: 986.

16. Toral JR, Martínez M, Llogel G, Peralba JI, Mellado del Rey F, Martos J. Lesiones hepáticas pseudometastásicas y linfadenitis necrotizante de Kikuchi. An Med Intern (Madrid) 1993; 10: 346-8.

17. Kikuchi M. Recurrence of Kikuchi's disease with aseptic meningitis in HTLV-1 carrier. [editorial; comment]. Intern Med 1996; 35: 753.

18. Kuipers EJ, Timens W, Daenen S. Kikuchi-Fujimoto disease complicated by severe rhabdomyolysis. Ann Hematol 1992; 65: 278-80.

19. Chan JKC, Wong KD, Ng CS. A fatal case of multicentric Kikuchi's histiocytic necrotizing lymphadenitis. Cancer 1989; 63: 1856-62.

20. Yen A, Fearneyhough P, Raimer SS, Hudnall SD. EBV-associated Kikuchi's histiocytic necrotizing lymphadenitis with cutaneous manifestations. J Am Acad Dermatol 1997; 36: 342-6.

21. Anagnostopoulos I, Humme M, Korbjuhn P, et al. Epstein-Barr virus in Kikuchi-Fujimoto disease [letter]. Lancet 1993; 341: 893.

22. Hollingsworth HC, Peiper SC, Weiss LM, Raffeld M, Jaffe ES. An investigation of the viral pathogenesis of Kikuchi-Fujimoto disease. Lack of evidence for Epstein Barr virus or human herpes virus type 6 as the causative agents. Arch Pathol Lab Med 1994; 118: 134-40.

23. Rodríguez JN, Mariano D, Elizalbe J y cols. Enfermedad de Kikuchi-Fujimoto asociada a infección aguda por virus herpes 6. Sangre 1996; 41 (5): 387-90.

24. Huh J, Hoon Kang G, Gong G, Sook Kim S, Ro JY and Woo Kim C. Kaposi's sarcoma-associated Herpesvirus in Kikuchi's disease. Human Pathol 1998; 29: 1091-6.

25. Bataille V, Harland CC, Behrens J, Cook MG, Holden CA. Kikuchi disease (histiocytic necrotizing lymphadenitis) in association with HTLV1. Br J Dermatol 1997; 136: 610-12.

26. Ereño C. Kikuchi lymphadenitis and AIDS [letter]. Histopathology 1999; 34: 273.

27. Pileri SA, Sabattini E, Costigliona P et al. Kikuchi's lymphadenitis and HIV infection [letter]. AIDS 1991; 5: 459-61.

28. Yufu Y, Matsumoto M, Miyamura T et al. Parvovirus B19 associated haemophagocytic syndrome with lymphadenopathy resembling histiocytic necrotizing lymphadenitis (Kikuchi's disease). Br J Haematol 1997; 96: 868-71.

29. Kikuchi M, Yoshizumi T, Nakamura H. Necrotizing lymphadenitis: possible acute toxoplasmic infection. Virchows Arch A Pathol Anat Histol 1977; 376: 247-53.
30. Heikens J, Tel W, Van de Stadt J, de Koning J, Ten Napel CH. Kikuchi's lymphadenitis: report of a Yersinia enterocolitica-associated case and an overview of aetiology and clinical outcome. Neth J Med 1992; 41: 222-8.

31. Rodríguez J, Martín MV, Báez JM, Gil JL. Linfadenitis necrotizante de Kikuchi-Fujimoto asociada a brucelosis. Sangre 1992; 37: 201-4.

32. Sumiyoshi Y, Kikuchi M, Takeshita M, Oshima K, Masuda Y. Alpha-interferon in Kikuchi's disease. Virchow Archiv B Cell Pathol 1991; 61: 201-7.

33. Kubota M, Tsukamoto R, Kurokawa K, Imai T and Furosho K. Elevated serum interferon $\mathrm{g}$ and interleukin-6 in patients with necrotizing lymphadenitis (Kikuchi's disease). Br J Haematol 1996; 95: 613-5.

34. Lima M, Silvestre F, Correia J, Oliveira J , Justica B. Kikuchi's disease: a case report with emphasis on flow cytometric studies. Sangre 1996; 41: 383-6.

35. Martínez-Vázquez C, Hughes G, Bordon J et al. Histiocytic necrotizing lymphadenitis, Kikuchi-Fujimoto's disease, associated with systemic lupus erythematosus. QJM 1997; 90: 531-3.

36. Eisner MD, Amory J, Mullaney B, Tierney Jr L, Browner WS. Necrotizing lymphadenitis associated with systemic lupus erythematosus. Semin Arthritis Rheum 1996; 26: 477-82.

37. Garazzi S, Grossin M, Kahr MF. Adult onset Still's disease and Kikuchi's disease. A new case [letter]. Rev Rhum Engl Ed 1997; 64: 352-3.

38. Otha A, Matsumoto Y, Otha T, Kaneoka H, Yamagushi M. Still's disease associated with necrotizing lymphadenitis (Kikuchi's disease): Report of 3 cases. J Rheumatol 1988; 15: 981-3.

39. Aqel NM, Amr SS, Najjar MM, Henry K. Kikuchi's lymphadenitis developing in a patient with mixed connective tissue disease and Hashimoto's tyroiditis [letter]. Br J Rheumatol 1997; 36: 1236-8.

40. Rubio SI, Plewinsky TS, Sabatin M, Poretsky L. Kikuchi's disease associated with Hashimoto's tyroiditis. J Endocrinol Invest 1996; 19: 136-7.

41. Sever CE, Leith CP, Apenzeller J, Foucar K. Kikuchi's necrotizing lymphadenitis associated with ruptured silicone breast implant. Arch Pathol Lab Med 1996; 120: 380-5.

42. Lazarus JH. Silent and subacute thyroiditis. In: The Thyroid: A fundamental and clinical text, 7th ed, Braverman, LE. Utiger, RD (Eds), Lippincott-Raven, Philadelphia 1996. p. 577.

43. Oristrell J , Miquel R. Mujer de 17 años con adenopatías y fiebre. Med Clin (Barc) 1999; 112: 428-435.

44. Snow RL, Ferry JA. A 24-year-old Asian woman with cervical lymphadenopathy and fever. Case records of the Massachusetts General Hospital (case 5-1997). N Engl J Med; 492-9.

45. Ferrer R. Lymphadenopathy: Differential diagnosis and evaluation. Am Fam Phys Physician 1998; 58: 1313.

46. Martínez Vázquez C, Vilariño C, Martínez-Cueto P, López A, Sopeña B, De La Fuente J. Linfadenitis necrotizante histiocitaria o enfermedad de Kikuchi. Apariencia radiológica mediante TC. An Med Interna (Madrid) 2002; 19: 183-5.

47. Tsang W, Chan J, Ng CS. Kikuchi's lymphadenitis. A morhologic analysis of 75 cases with special reference to unusual features. Am J Surg Pathol 1994; 18: 219-31.

48. Imamura M, Matsuura A, Kamiya H, Suzuki T, Kikuchi K, Onoe T. An ultrastructural study of subacute necrotizing lymphadenitis.Am J Pathol 1982; 107: 292-9.

49. Menasce LP, Banerjee SS, Edmonson D, Harris M. Histiocytic necrotizing lymphadenitis (Kikuchi-Fujimoto's disease): continuing diagnostic difficulties. Histopathology 1998; 33: 248-54.

50. Iguchi H, Sunami K, Yamane H et al. Apoptotic cell death in Kikuchi's disease: a TEM study. Acta Otolaryngol 1998; (Supl.) 538: 250-3.

51. Turner RR, Martín J, Dorfman RF. Necrotizing lymphadenitis, a study of 30 cases. Am J Surg Pathol 1983; 7: 115-23.

52. Chamulak GA, Brynes R, Nathwani BN. Kikuchi-Fujimoto disease mimicking malignant lymphoma. Am J Surg Pathol 1990; 14: 514-23.

53. Smith KGC, Becker GJ, Busmanis I. Recurrent Kikuchi's disease [letter]. Lancet 1992; 340:124.

54. Lin SH, Ko WS, Lee HS, Hwang WS. Kikuchi's disease associated with lupus-like syndrome: a fatal case. J Rheumatol 1992; 19: 1995-6.

55. Tsai MK, Huang HF, Hu RH et al. Fatal Kikuchi-Fujimoto disease in trasplant recipients: a case report. Transplant Proc 1998; 30: 3137-8.

56. O'Neill D, O'Grady J, Variend S. Child fatality associated with pathological features of histiocytic necrotizing lymphadenitis (Kikuchi-Fujimoto disease). Pediatr Pathol Lab Med 1998; 18 (1): 79-80. 